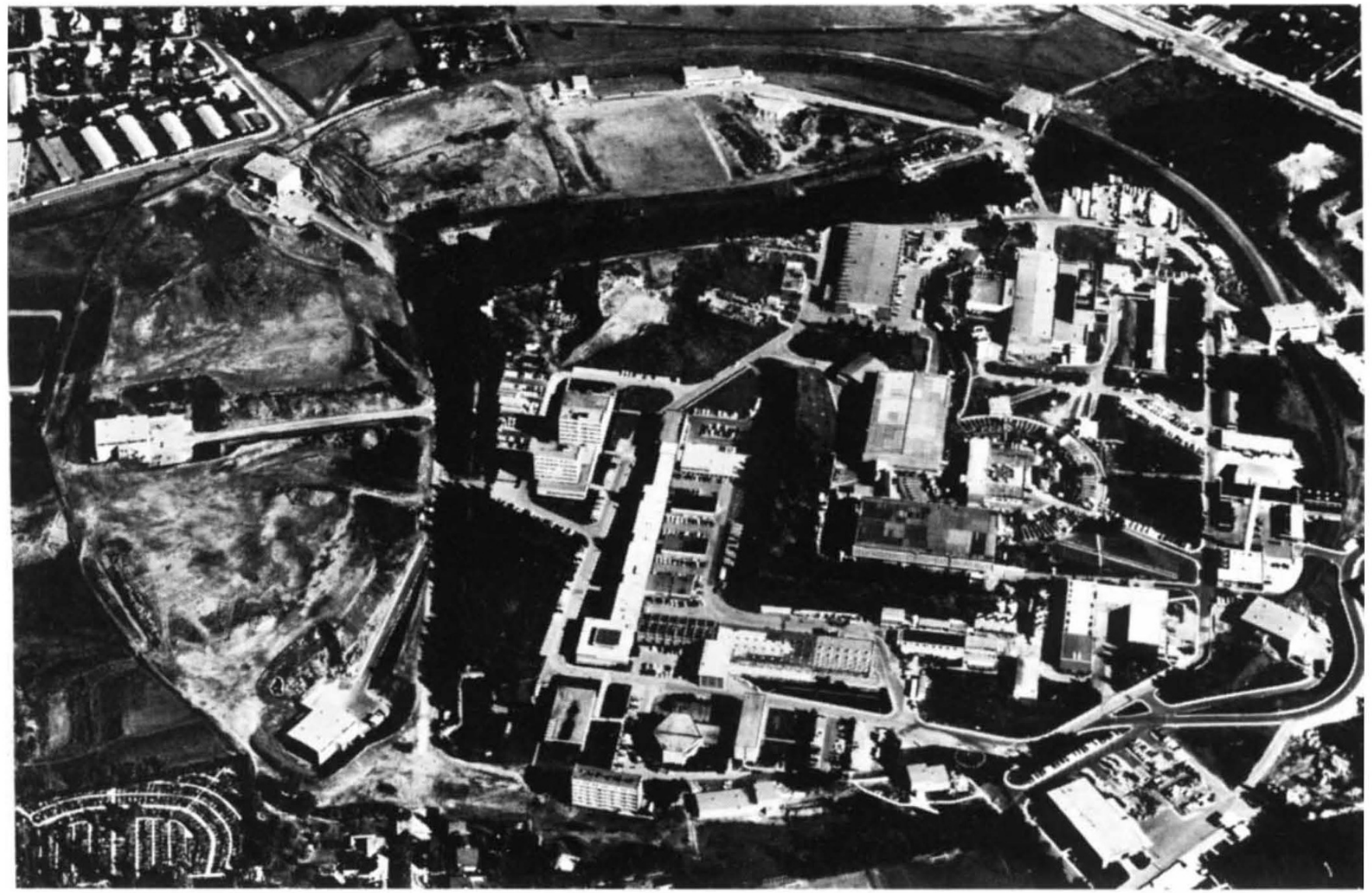

\title{
PETRA puts European physics in the lead
}

PETRA, Europe's $19 \mathrm{GeV}$ on $19 \mathrm{GeV}$ electron-positron storage ring under construction at the Deutsches Elektronen Synchrotron laboratory (DESY) near Hamburg, has circulated its first beam around the ring and stored it "for several minutes".

At $10 \mathrm{pm}$ on Saturday night, 15 July, a team of accelerator physicists headed by Dr Gustav-Adolf Voss successfully injected a beam of electrons along the axis into PETRA, and allowed it to circulate without any optical correcting magnets (sextupoles) switched on. Monitors detected no observable decay of the beam, but the beam was killed before a measurement of its lifetime could be made. This puts PETRA at least a year ahead of PEP, its American rival.

PETRA also won a race for funds within Europe against a British version of the same machine, EPIC, which was to have been built at the Rutherford Laboratory in Berkshire. It has been built with great speed and great efficiency by the German physicists, who seem to have adopted something of an Amcrican style to get the accelerator underway.

PETRA costs have been trimmed right down, and the machine has few frills-in contrast to its $10 \mathrm{GeV}$ on $10 \mathrm{GeV}$ predecessor, DORIS, which was arguably over-sophisticated.
DORIS ran into troubles in its commissioning, ending up with a much lower beam intensity - and hence data rate-than had been expected. Thus it was the American counterpart to DORIS, SPEAR at Stanford, California, that stole the limelight in the Nobel Prize winning discovery of charm, although DORIS ultimately made some extremely useful contributions.

With PETRA the boot seems to be on the other foot. If there is good physics to be discovered at these energies it seems likely that PETRA will discover it, rather than the American PEP, which will be left to play an important but supporting role. In conjunction with CERN's $400 \mathrm{GeV}$ super proton synchrotron at Geneva, with its forthcoming high intensity lepton beams, Europe now seems set to take the lead from America in the high energy physics of leptons, which is where, in recent years, the most important discoveries of physics have come.

The next step at PETRA will be to make detailed measurements of the beam optics, and bring in the correcting sextupoles. The last obstacle to producing beam for experiments is likely to be the experiments themselves, the first of which is not likely to be ready before mid-September. This experiment
-PLUTO, a large magnetic detector working much like a big electronic bubble chamber surrounding a collision region-will begin to take data at around $10 \mathrm{GeV}$ to establish an overlap with the previous machine, DORIS, and then go straight to the highest energies $(38 \mathrm{GeV})$ to measure the total cross section for producing hadrons, divided by that for muon pairs. This quantity, $R$, made famous by experiments on charm, will tell the physicists how much interesting physics there is in between 10 and $38 \mathrm{GeV}$. The higher is $R$, the more physics-like new quarks and new leptons-there will be for PETRA to discover.

For the moment there will be a gap of a few weeks while DORIS, the old storage ring system which is involved in the injection of electrons into PETRA, is used for an experiment on its own: a search for the "upsilon prime" at a mass of over $10 \mathrm{GeV}$. The 9.5 GeV "upsilon", discoverce by Lcon Lederman last year and reconfirmed by DORIS recently, is believed to be a state consisting of a new quark ("bottom") and its antiquark (analogous to the $\mathrm{J} / \mathrm{psi}$ containing charmed and anticharmed quarks). The observation of the upsilon prime (an excited state of the upsilon) would help to confirm that interpretation.

Robert Walgate 